\title{
Design of a local quasi-distributed tuning and matching circuit for dissolution DNP cross polarization
}

Vinther, Joachim Møllesøe; Zhurbenko, Vitaliy; Albannay, Mohammed M.; Ardenkjær-Larsen, Jan Henrik

Published in:

Solid State Nuclear Magnetic Resonance

Link to article, DOI:

10.1016/j.ssnmr.2019.04.006

Publication date:

2019

Document Version

Peer reviewed version

Link back to DTU Orbit

Citation (APA):

Vinther, J. M., Zhurbenko, V., Albannay, M. M., \& Ardenkjær-Larsen, J. H. (2019). Design of a local quasidistributed tuning and matching circuit for dissolution DNP cross polarization. Solid State Nuclear Magnetic Resonance, 102, 12-20. https://doi.org/10.1016/j.ssnmr.2019.04.006

\section{General rights}

Copyright and moral rights for the publications made accessible in the public portal are retained by the authors and/or other copyright owners and it is a condition of accessing publications that users recognise and abide by the legal requirements associated with these rights.

- Users may download and print one copy of any publication from the public portal for the purpose of private study or research.

- You may not further distribute the material or use it for any profit-making activity or commercial gain

- You may freely distribute the URL identifying the publication in the public portal 


\section{Accepted Manuscript}

Design of a local quasi-distributed tuning and matching circuit for dissolution DNP cross polarization

JoachimM.O. Vinther, Vitaliy Zhurbenko, Mohammed M. Albannay, Jan Henrik Ardenkjær-Larsen

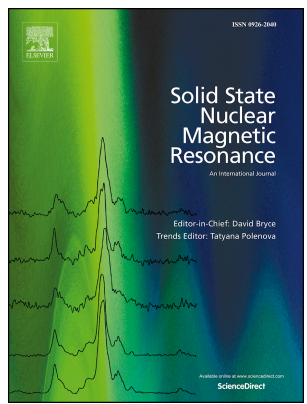

PII:

S0926-2040(19)30014-1

DOI:

https://doi.org/10.1016/j.ssnmr.2019.04.006

Reference: $\quad$ YSNMR 1598

To appear in: Solid State Nuclear Magnetic Resonance

Received Date: 3 February 2019

Revised Date: 3 April 2019

Accepted Date: 19 April 2019

Please cite this article as: J.O. Vinther, V. Zhurbenko, M.M. Albannay, J.H. Ardenkjær-Larsen, Design of a local quasi-distributed tuning and matching circuit for dissolution DNP cross polarization, Solid State Nuclear Magnetic Resonance (2019), doi: https://doi.org/10.1016/j.ssnmr.2019.04.006.

This is a PDF file of an unedited manuscript that has been accepted for publication. As a service to our customers we are providing this early version of the manuscript. The manuscript will undergo copyediting, typesetting, and review of the resulting proof before it is published in its final form. Please note that during the production process errors may be discovered which could affect the content, and all legal disclaimers that apply to the journal pertain. 


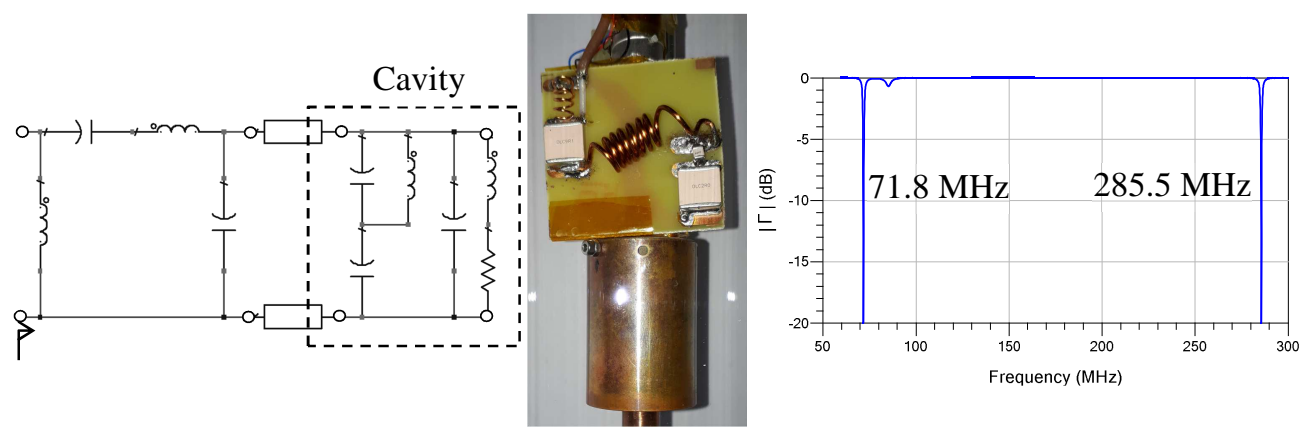



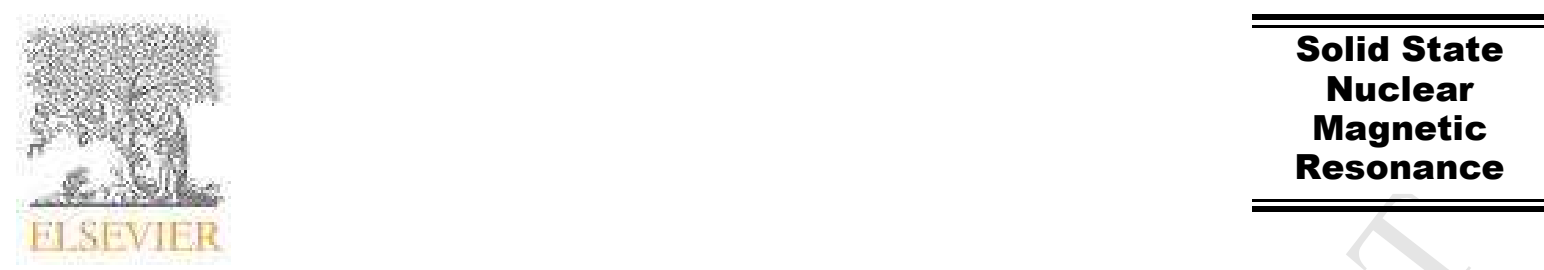

\title{
Design of a local quasi-distributed tuning and matching circuit for dissolution DNP cross polarization
}

\author{
Joachim M. O. Vinther ${ }^{\mathrm{a}, \#}$, Vitaliy Zhurbenko ${ }^{\mathrm{b}, \#}$, Mohammed M. Albannay ${ }^{\mathrm{a}}$, Jan Henrik \\ Ardenkjær-Larsen $^{\mathrm{a}^{*}}$ \\ ${ }^{a}$ Center for Hyperpolarization in Magnetic Resonance, Magnetic Resonance, Department of Health Technology, Technical University of \\ Denmark, Ørsteds Plads, 2800, Kgs. Lyngby, Denmark \\ ${ }^{b}$ Center for Hyperpolarization in Magnetic Resonance, Electromagnetic Systems Group, Department of Electrical Engineering, Technical \\ University of Denmark, Ørsteds Plads, 2800, Kgs. Lyngby, Denmark
}

Elsevier use only: Received date here; revised date here; accepted date here

\begin{abstract}
Dynamic nuclear polarization (DNP) build-up times at low temperature for low-gamma heteronuclei can be unfavorably long and can be accelerated by transfer of polarization from protons. The efficiency of the cross polarization (CP) depends on the $\mathrm{B}_{1}$-field strengths, the pulse sequence chosen for cross polarization and the sample composition. $\mathrm{CP}$ experiments rely on high $\mathrm{B}_{1}$-fields, which typically lead to electrical discharge and breakdown in the circuit. This problem is particularly severe in the low pressure helium atmosphere due to easily ionized helium atoms. The purpose of this study is to identify strategies to minimize voltages across components in a tuning and matching circuit of the coil to avoid electrical discharge during $\mathrm{CP}$ experiments. Design equations for three tuning and matching network configurations are derived. The results of the study are then used in the design of a single coil double resonance DNP probe operating at $71.8 \mathrm{MHz}\left({ }^{13} \mathrm{C}\right.$ frequency) and $285.5 \mathrm{MHz}$ ( ${ }^{1} \mathrm{H}$ frequency). In the current setup we achieve $28 \%$ polarization on ${ }^{13} \mathrm{C}$ in urea with a build-up time of 11.6 min with $\mathrm{CP}$ compared to $14 \%$ and $53 \mathrm{~min}$ by direct polarization using TEMPOL as the radical. Different cross polarization sequences are compared.
\end{abstract}

Keywords: electrical discharge, helium, DNP probe, matching circuit, cross-polarisation, CP

\#These authors contributed equally to this manuscript and should be considered co-first authors.
${ }^{*}$ Corresponding author. Tel.: +0-000-000-0000 ; fax: +0-000-000-0000 ; e-mail: author@institute.xxx . 


\section{Introduction}

Static DNP at low temperature, e.g. below $4 \mathrm{~K}$, is slow, and build-up time constants for low-gamma nuclei such as ${ }^{13} \mathrm{C}$ are typically $1000 \mathrm{~s}$ in favorable samples such as neat pyruvic acid [1], or longer. For dilute low-gamma nuclei the build-up time can be prohibitively long. Schemes for faster DNP polarization build-up on these types of samples are therefore warranted. A possibility is to go through faster polarizing proton spins, and cross polarize the low-gamma nuclei. However, fast and high proton polarization typically requires some sample optimization, e.g. favoring diluting the proton concentration significantly. Thus, in vivo applications of dissolution DNP (dDNP) where the sample size can be up to several grams for humans, and the concentration of both protons and low-gamma nuclei $\left({ }^{13} \mathrm{C}\right)$ is often very high $(10-100 \mathrm{M})$, the benefit of cross-polarization may be limited as the build-up can be optimized by other means such as choice of radical or microwave modulation [2]. However, in the case of in vitro samples with low concentration of solute and dilute nuclear spins (e.g. [3]), cross polarization may be the preferred and only option to achieve acceptable low-gamma nuclear DNP.

$\mathrm{CP}$ experiments require high amplitude radiofrequency (RF) pulses typically $>30 \mathrm{kHz}$ for Hartmann-Hahn CP [4], which is demanding especially for the heteronucleus. Attaining these high RF pulses proves challenging due to the imposed coil geometry by the requirements for dissolution capability and the inherent low breakdown voltage of the gaseous helium atmosphere above the liquid helium in a cryostat cooled to ca. $1 \mathrm{~K}$. A few realizations have yet been published:

A locally, double tuned and matched solenoid with a $\mathrm{B}_{1}$-field strengths of $55 \mathrm{kHz}$ (corresponding nutation frequency) yielding $20 \%{ }^{13} \mathrm{C}$ polarization after CP was reported [5].

A remotely, double tuned and matched saddle coil with five windings located outside the microwave cavity presented $\mathrm{B}_{1}$-field strengths of $30 \mathrm{kHz}$, resulting in a two-fold improvement when comparing $\mathrm{CP}$ and direct DNP [6].

A dissolution-capable system consisting of two, inductively coupled, locally tuned and matched coils, yielding $\mathrm{B}_{1}$-field strengths of $20 \mathrm{kHz}$. Combined with gated microwaves and successive $\mathrm{CP}$ transfers over $70 \%{ }^{13} \mathrm{C}$ polarization is reported [7].

A remotely, double tuned and matched solenoid capable of $B_{1}$-field strengths of $100 \mathrm{kHz}$, but without dissolution capabilities yielded a CP enhancement of a factor of 2.5 [8].

Similar enhancements were presented earlier in the thesis by Pérez Linde for solenoid $(50 \mathrm{kHz} \mathrm{B})_{1}$ and saddle coils comparing different $\mathrm{CP}$ sequences [9].

The $\mathrm{B}_{1}$-field strengths achievable are proportional to the square root of power that can be applied, which is often limited by electrical discharge and breakdown due to ionization of helium atoms surrounding the cryo-cooled circuit. This ionization is caused by high electrical potentials in the circuit. The problem is especially severe in low loss resonant circuits, where voltage amplitudes can be much higher than the voltage of the input signal. These circuits are typically formed by capacitors of the tuning and matching network, which resonate with the inductance of the coil. In Sec. 2 of this study, we analyze voltages across matching and tuning components in order to identify strategies to reduce voltages in the circuit. Following these strategies, a double tuned and matched resonance circuit for $\mathrm{CP}$ is developed and described in Sec. 3. Sec. 4 details the materials and methods for the experimental work. The experimental realization of the double-resonance probe was subjected to tests of electrical discharge limitations, $\mathrm{B}_{1}$-field strength capabilities and crosspolarization efficiency of different $\mathrm{CP}$ sequences. Moreover, a study of the dependence of $\mathrm{CP}$ efficiency on sample protonation degree is presented. These experimental results are summarized in Sec. 5. 


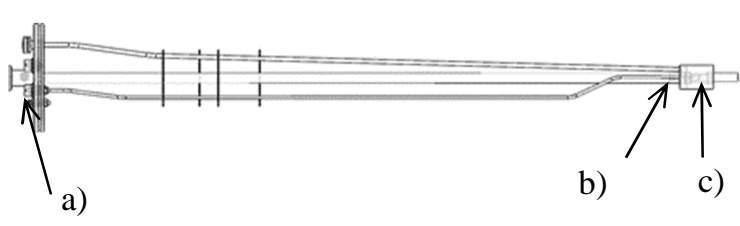

Figure 1. Dissolution DNP probe and different placement of RF tuning and matching circuit: a) remote, b) remote outside cavity, and c) local on coil.

One of the considerations in DNP probe design is where to place the matching and tuning network with regards to the sample coil. The circuit can be placed at the flange of the probe, position a) in Figure 1. This is the most convenient location since the sample coil can be matched and tuned to any sample or nuclei without removing the probe from the cryostat. That is, however, a very inefficient setup due to high loss in the coaxial cable connecting the tuning and matching network with the coil. A significant part of these losses can be attributed to standing waves in the cable [10].

To avoid cable loss, the tuning and matching circuit should be placed right at the coil, position c) in Figure 1. However, often a limited cavity space renders this solution challenging. Therefore, a good compromise could be to place a part of the tuning and matching network right outside the cavity at position b) in Figure 1). We denote this shared location as quasi-distributed, i.e., with tuning and matching components distributed between position b) and c) in Figure 1. This part of the DNP probe is in liquid helium at low pressure, which makes the circuits susceptive to electrical discharge even at relatively low transmit powers, presumably due to occasional occurrence of gaseous helium at low pressure. Figure 2 shows a photograph of such an event, which is also observed as a dramatic increase in reflected power. In the following Section we investigate strategies to avoid such effects by minimizing voltage amplitudes in the tuning and matching circuit.

\section{Voltage amplitudes in a tuning and matching circuit}

First, we consider several configurations for coil tuning and matching (when the circuit is placed in position b) or c) in Figure 1) and analyze resulting

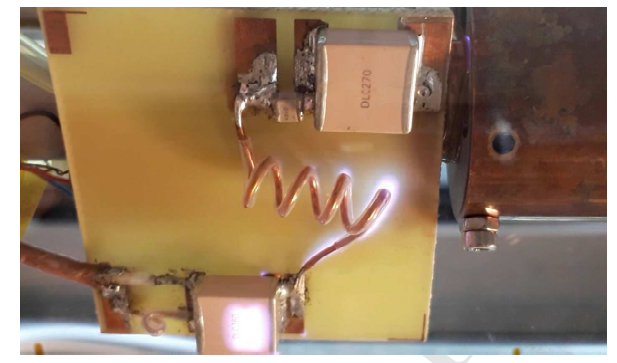

Figure 2. Photograph of electrical discharge in low pressure helium atmosphere across matching network components during transmit pulse.

voltages. Then, a double resonance matching circuit design is presented.

\subsection{Voltages in a conventional tuning and matching circuit}

In considerations below, we assume that the coil can be equivalently represented as an inductor $L$ in series with a resistor $R$, as shown in Figure 3. $L$ is then an inductance of the coil and $R$ represents the loss in the conductor of the coil as well as loss due to coupling to the sample.

\section{Case 1}

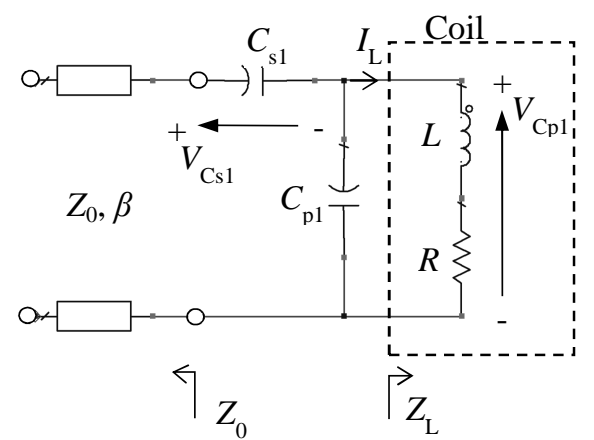

Figure 3. Case 1: coil with a tuning and matching circuit connected to a transmission line.

Different types of matching circuits can be used to transform impedance of the coil, $Z_{\mathrm{L}}=R+j \omega L$, to a system impedance $\mathrm{Z}_{0}$ (usually $50 \Omega$ ). Here, $\omega=2 \pi f$ is an operating frequency and $j=\sqrt{-1}$. Often, a circuit consisting of a parallel capacitor $C_{\mathrm{p} 1}$ and series capacitor $C_{\mathrm{s} 1}$ (refer to Figure 3 ) is used for coil tuning and matching due to its simplicity and availability of 
the required capacitor values (it should be kept in mind of course, that such a simple circuit cannot match a full possible range of impedances $Z_{\mathrm{L}}$ [11]).

The capacitance values can be expressed through corresponding susceptance $\mathfrak{B}_{1}$ and reactance $X_{1}$ :

$$
\begin{aligned}
& C_{p 1}=\frac{\mathfrak{B}_{1}}{\omega}, \\
& C_{s 1}=\frac{-1}{X_{1} \omega},
\end{aligned}
$$

where $\mathfrak{B}_{1}$ and $X_{1}$ are found from matching conditions [12]:

$$
\begin{aligned}
& \mathfrak{B}_{1}=\frac{\omega L \pm \sqrt{\frac{R}{Z_{0}}} \sqrt{R\left(R-Z_{0}\right)+(\omega L)^{2}}}{R^{2}+(\omega L)^{2}}, \\
& X_{1}=\frac{1}{\mathfrak{B}_{1}}+\frac{\omega L Z_{0}}{R}-\frac{Z_{0}}{\mathfrak{B}_{1} R} .
\end{aligned}
$$

Obviously, the equations (1) and (2) are valid when $\mathfrak{B}_{1}$ and $X_{1}$ are positive, therefore an appropriate sign in eq. (3) should be chosen. In addition, $\mathfrak{B}_{1}$ must be real, which sets the range of possible $Z_{\mathrm{L}}$.

The voltages in the circuit can then be found using basic circuit theory:

$$
\begin{aligned}
& V_{C p 1}=I_{L} Z_{L}, \\
& V_{C s 1}=V_{C p 1} X_{1} \frac{j-B_{1} Z_{L}}{Z_{L}} .
\end{aligned}
$$

The magnetic field in a simple loop coil is directly proportional to the coil current $I_{\mathrm{L}}$. As follows from Ohm's law, eq. (5), for the given current, the voltage across the coil and parallel capacitor, $V_{\mathrm{Cp} 1}$, is directly proportional to the impedance of the coil. From here, in order to reduce the voltage amplitude across the parallel capacitor one should strive for lower magnitude of the coil impedance $\left|Z_{\mathrm{L}}\right|$. Since coils are usually designed for high efficiency, the reactive part of the impedance is much larger than the resistive part. Therefore, it is preferable to minimize the inductance of the coil to minimize the corresponding voltage drop. The voltage amplitudes $\left|V_{\mathrm{Cp} 1}\right|$ and $\left|V_{\mathrm{Cs} 1}\right|$ in the circuit for a range of magnitudes of coil impedance is shown in Figure 4.

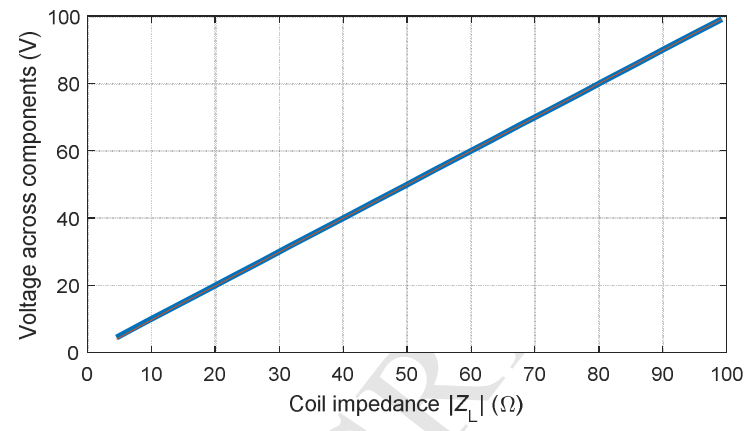

Figure 4. Voltage amplitudes in the circuit in Figure 3 for a current of $1 \mathrm{~A}$ in the coil $L$. Here $\left|\mathrm{V}_{\mathrm{Cp} 1}\right| \approx\left|\mathrm{V}_{\mathrm{Cs} 1}\right|$.

The plot in Figure 4 is for $R=0.1 \Omega$ and a range of reactances to $100 \Omega$. For example, for $71.8 \mathrm{MHz}$, which is ${ }^{13} \mathrm{C}$ resonance frequency at $6.7 \mathrm{~T}$ field, this range corresponds to inductance range of approximately from $10 \mathrm{nH}$ to $220 \mathrm{nH}$.

The voltages in the circuit for a range of coil resistance $R$ and fixed $L=20 \mathrm{nH}$ is shown in Figure 5 .

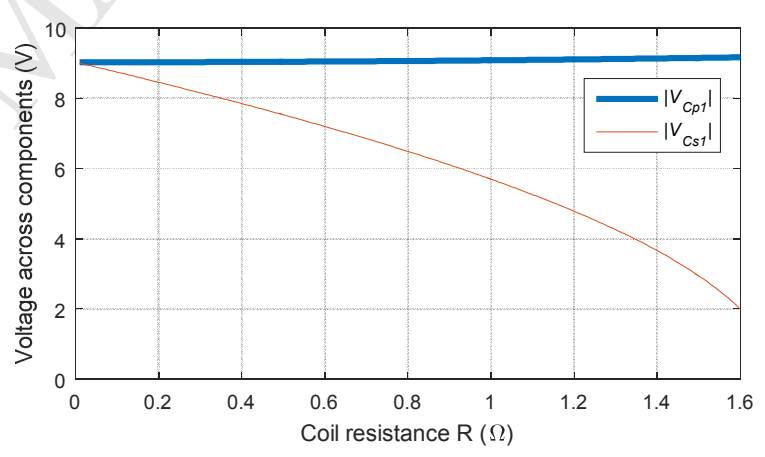

Figure 5. Voltages in the circuit in Figure 3 for 1A current in the $\operatorname{coil} L$.

As can be seen, the voltage amplitude across the series capacitor, $\left|V_{\mathrm{Cs} 1}\right|$, reduces with higher loss in the coil, while $\left|V_{\mathrm{Cp1}}\right|$ is not affected. This is expected, since a lower transformation ratio is required from the circuit resulting in less extreme reactances.

\subsection{Remote (outside cavity) and quasi-distributed matching schemes}

Placing the RF circuit next to the coil is optimal from both a voltage and efficiency point of view [10]. The efficiency of remote tuning and matching is 
significantly affected by loss in the transmission line, which becomes comparable to the loss of the coil itself, even for low loss lines. It is, however, not always possible nor convenient to place the RF circuit right at the coil. Often, a remote configuration has to be used, i.e., a tuning and matching circuit connected to the coil through a transmission line. In this section we consider quasi-distributed (Figure 6.a) and remote (outside cavity - Figure 6.b) tuning and matching circuits.

We start with the analysis of the first circuit in Figure 6.a. The capacitance values can be expressed through corresponding susceptance $\mathfrak{B}_{2}$ and reactance $X_{2}$ :

$$
\begin{aligned}
& C_{p 2}=\frac{\mathfrak{B}_{2}}{\omega}, \\
& C_{s 2}=\frac{-1}{X_{2} \omega},
\end{aligned}
$$

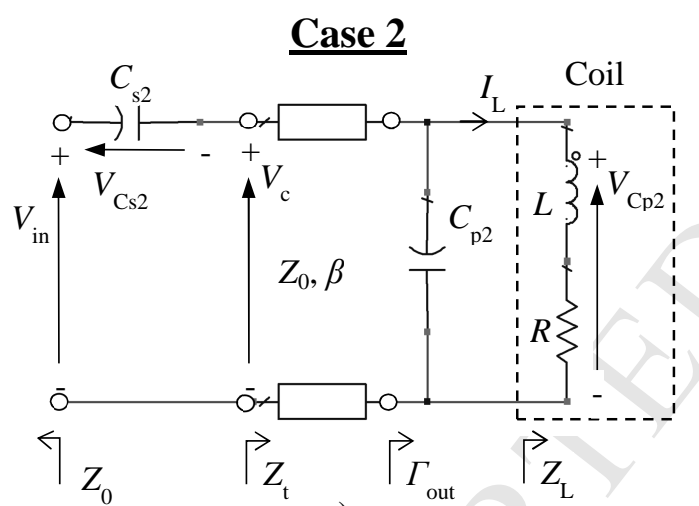

a)

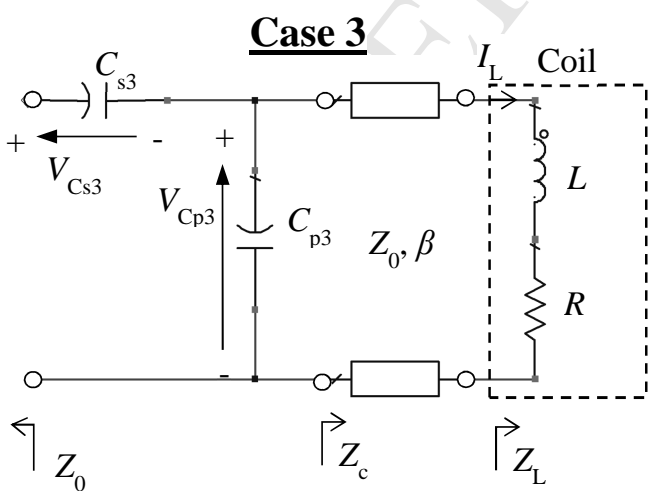

b)

Figure 6. a) Case2 - quasi-distributed and b) Case 3 - remote (outside cavity) tuning and matching of a coil where $\mathfrak{B}_{2}$ and $X_{2}$ are found from matching conditions. Assuming $Z_{0}=50$ and lossless transmission line (refer to Appendix A):

$$
\mathfrak{B}_{2}=\frac{R+\omega L \tan (\beta l)+\frac{a \sqrt{R} \tan (\beta l)}{10}-50}{50(R \tan (\beta l)-\omega L)-5 a \sqrt{R}}
$$

where $a=\sqrt{2} \sqrt{\left(R^{2}+\omega L^{2}\right)\left(\tan ^{2}(\beta l)+1\right)-50 R}+$ $10 \sqrt{R} \tan (\beta l)$ is an auxiliary variable, and

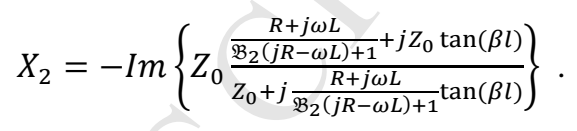

Here, $l$ is the length and $\beta=2 \pi / \lambda$ is the phase constant of the transmission line. $\lambda$ is the guided wavelength in the transmission line. Obviously, $\mathfrak{B}_{2}$ and $X_{2}$ has to be positive in order for equations (7) and (8) to be valid. Here we neglect loss in the transmission line to simplify the equations. This loss will of course have impact on voltage amplitude, but the general trend will not change.

The voltage across the parallel capacitor is expressed in the similar way as for the Case 1 in Figure 3 (eq.(5)):

$$
V_{C p 2}=I_{L} Z_{L}
$$

and the voltage across the series capacitor

$$
V_{C s 2}=V_{\text {in }}-V_{c}=\frac{j V_{C p 2}\left(e^{j \beta l}+\Gamma_{o u t} e^{-j \beta l}\right)}{\left(1+\Gamma_{o u t}\right)\left(B_{2} Z_{0}-j\right)},
$$

where

$$
\Gamma_{\text {out }}=\frac{Z_{L}-Z_{0}\left(j \mathfrak{B}_{2} Z_{L}+1\right)}{Z_{L}+Z_{0}\left(j \mathfrak{B}_{2} Z_{L}+1\right)} .
$$

The analysis of Case 3 in Figure 6.b is similar to Case 1. The capacitance values can be expressed through corresponding susceptance $\mathfrak{B}_{3}$ and reactance $X_{3}$ :

$$
\begin{aligned}
& C_{p 3}=\frac{\mathfrak{B}_{3}}{\omega}, \\
& C_{s 3}=\frac{-1}{X_{3} \omega},
\end{aligned}
$$


where $\mathfrak{B}_{3}$ and $X_{3}$ are found from matching conditions:

$$
\begin{gathered}
\mathfrak{B}_{3}=\frac{\operatorname{Im}\left\{Z_{c}\right\} \pm \sqrt{\frac{\operatorname{Re}\left\{Z_{c}\right\}}{Z_{0}}} \sqrt{\operatorname{Re}\left\{Z_{c}\right\}\left(\operatorname{Re}\left\{Z_{c}\right\}-Z_{0}\right)+\left(\operatorname{Im}\left\{Z_{c}\right\}\right)^{2}}}{\left(\operatorname{Re}\left\{Z_{c}\right\}\right)^{2}+\left(\operatorname{Im}\left\{Z_{c}\right\}\right)^{2}} \\
X_{3}=\frac{1}{\mathfrak{B}_{3}}+\frac{Z_{0} \operatorname{Im}\left\{Z_{c}\right\}}{\operatorname{Re}\left\{Z_{c}\right\}}-\frac{Z_{0}}{\mathfrak{B}_{3} \operatorname{Re}\left\{Z_{c}\right\}}
\end{gathered}
$$

where

$$
Z_{c}=Z_{0} \frac{Z_{L}+j Z_{0} \tan (\beta l)}{Z_{0}+j Z_{L} \tan (\beta l)}
$$

is the input impedance of the transmission line loaded with $Z_{\mathrm{L}}$ [13].

The equations (14) and (15) are valid when $\mathfrak{B}_{3}$ and $X_{3}$ are positive, therefore an appropriate sign in eq. (16) should be chosen. In addition, $\mathfrak{B}_{3}$ must be real.

The voltage across the parallel capacitor in Case 3 becomes

$$
V_{C p 3}=\frac{I_{L} Z_{L}\left(e^{j \beta l}+\Gamma_{L} e^{-j \beta l}\right)}{\left(1+\Gamma_{L}\right)}
$$

where

$$
\Gamma_{L}=\frac{Z_{L}-Z_{0}}{Z_{L}+Z_{0}}
$$

is the reflection coefficient from the coil $Z_{\mathrm{L}}$.

The voltage across the series capacitor is expressed in a similar way as for Case 1 in Figure 3 (eq. (6)):

$$
V_{C s 3}=V_{C p 3} X_{3} \frac{j-B_{3} Z_{c}}{z_{c}}
$$

Now we can compare voltages in the circuits for all three cases. The results for a range of transmission line electrical lengths are shown in Figure 7 and Figure 8. The plots are for $R=0.1 \Omega$ and $\omega L=2 \pi f L$ $=10 \Omega$, that corresponds to $L \approx 22 \mathrm{nH}$ at $f=$ $71.8 \mathrm{MHz}$. At this frequency, the electrical length of $0.2 \lambda$ corresponds to almost $60 \mathrm{~cm}$ coaxial cable filled with Teflon.

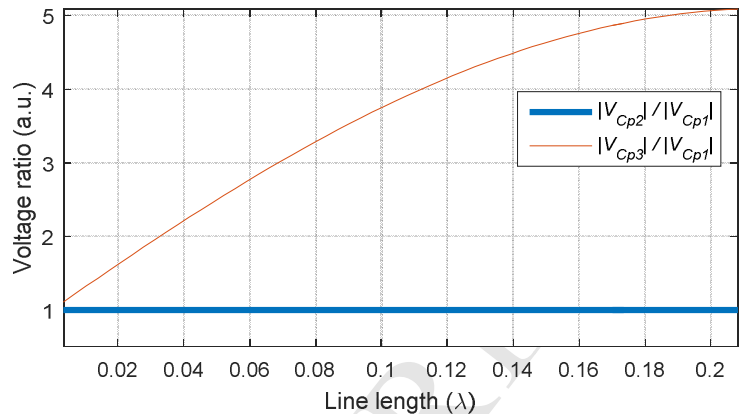

Figure 7. Comparison of voltages across parallel capacitor for three cases illustrated in Figure 3 and Figure 6 for a fixed current in the coil.

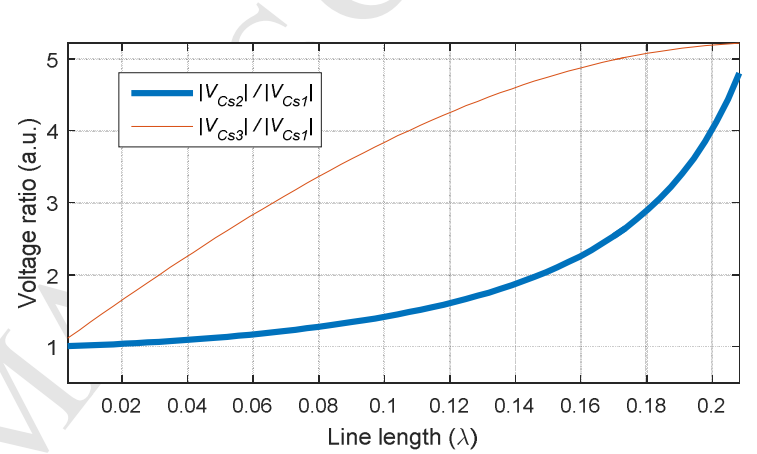

Figure 8. Comparison of voltages across series capacitor for three cases illustrated in Figure 3 and Figure 6 for a fixed current in the coil.

As equation (11) suggests (and illustrated in Figure 7), the quasi-distributed matching scheme (Case 2, Figure 6.a) offers the same voltage drop across the parallel capacitor as local matching (Case 1, Figure 3.). This is also true for the series capacitor if the transmission line is kept short. On the other hand, voltages in the remote matching circuit (Case 3 in Figure 6.b.) grow relatively fast with the transmission line length. For example, $4.5 \mathrm{~cm}$ cable in remote matching scheme results in almost $50 \%$ increase in voltage amplitude across the capacitors in comparison to local matching for the chosen coil reactance. It should be noted, that the relative voltage grows faster with $l$ for lower coil reactances.

From the results in Figure 7 and Figure 8 it seems that a quasi-distributed tuning and matching circuit could be a good compromise between component placement flexibility and voltage amplitudes. 


\subsection{Bandwidth comparison}

The bandwidth of the RF-circuit is indicative of how sensitive the circuit is to the values of the components and, eventually, how difficult it is to fine-tune the circuit in practice. To compare bandwidths, we plot the simulated reflection coefficient magnitude for the circuits in Figure 3 and Figure 6, which were designed using equations (1), (2), (7), (8), (14), and (15). The results for a transmission line length of $0.1 \lambda$ are shown in Figure 9.

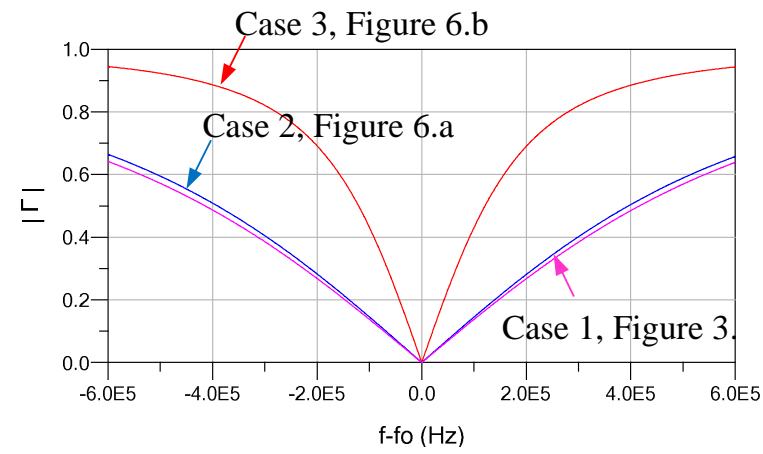

Figure 9. Magnitude of the reflection coefficient at the input terminals of the circuits in Figure 3 and Figure $6 . \mathrm{f}_{0}$ is the operating frequency of the coil.

The transmission line contributes to the reactance in the circuit, which results in a narrower bandwidth for the remote matching scheme. It should be kept in mind that the considered transmission line is lossless and introducing transmission line loss can have a considerable impact on bandwidth. Yet, what can be concluded from the data in Figure 9, is that the quasidistributed scheme (Case 2, Figure 6.a) provides almost the same bandwidth as the local tuning and matching scheme (Case 1, Figure 3) and consequently, similar sensitivity to the circuit component tolerances.

\section{Double resonance circuit design}

$\mathrm{CP}$ experiments require magnetic fields at ${ }^{13} \mathrm{C}$ and ${ }^{1} \mathrm{H}$ resonance frequencies. Those could be generated by two orthogonal coils with two matching circuits and two transmission lines [14]. Alternatively, the fields could be generated by a single coil connected to a single transmission line through a double resonance tuning and matching circuit. This approach has been exploited in this work using an AldermanGrant coil shown in Figure 10: A copper tube section $(\mathrm{OD}=15 \mathrm{~mm}, \mathrm{ID}=13 \mathrm{~mm}, \mathrm{RRR}>50)$ was cut to length
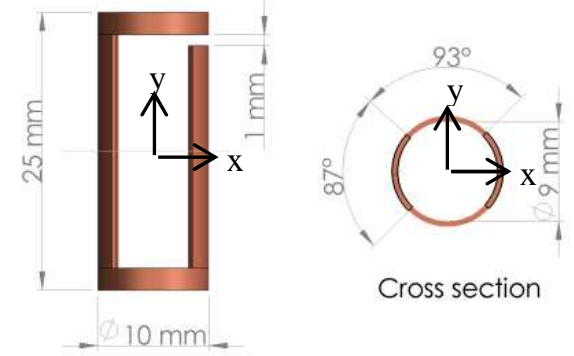

Figure 10. The Alderman-Grant coil used for the double-tuned circuit. The on-coil capacitors are placed at and across the gap.

to determine the height of the coil. Both $87^{\circ}$ slots and excitation slit were subsequently milled to form the final coil geometry.

The coil has $95 \%$ homogeneity over a region of 8 $\mathrm{mm}$ DSV (diameter of sphere volume). Full wave electromagnetic analysis of the coil results in the magnetic field distribution shown in Figure 11 with $\mathrm{L} \approx 15 \mathrm{nH}$ and $R_{71.8 \mathrm{MHz}} \approx 19 \mathrm{~m} \Omega ; R_{285.5 \mathrm{MHz}} \approx 40 \mathrm{~m} \Omega$. The advantage of the single coil is that both frequencies have the highest possible magnetic field at unit current (smallest size) and a single transmission line can be used to minimize heat loads to the helium bath. 

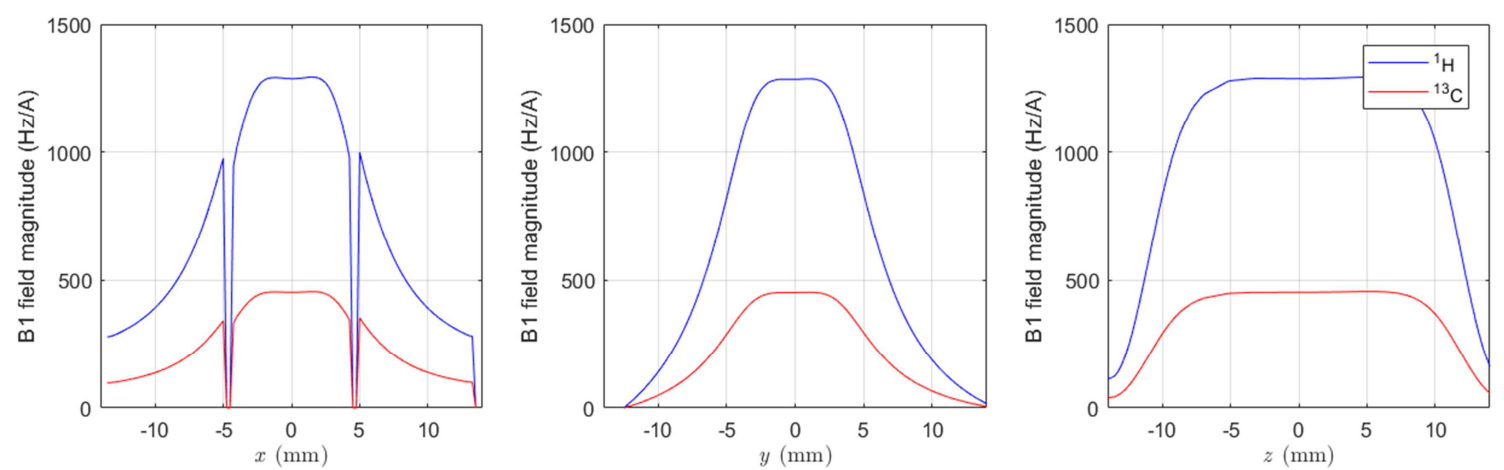

Figure 11. Simulated spatial distribution of the magnetic field amplitude for the AG coil expressed as nutation frequency equivalents per unit current. Axes titles refer to Figure 10.

For $\mathrm{CP}$, we design a double resonance tuning and matching circuit. Following the approach in the previous section, the parallel components are placed directly on the coil in the cavity, in order to minimize the voltages in the circuit. The remaining components are placed outside the cavity using a $2.5 \mathrm{~cm}$ long 50 $\Omega$ coaxial cable. The schematic of the circuit is shown in Figure 12. Due to a high transformation ratio ( $R$ to $50 \Omega$ ), the circuit is narrowband. For accurate performance prediction, the complete circuit model should include parasitics of the interconnects and implemented components. In order not to obscure the model, those parasitics are omitted in Figure 12. The inductance and loss in the coil are represented by $L$ and $R . C_{1}$ is chosen to resonate with $L$ close to ${ }^{1} \mathrm{H}$ frequency. The parallel $L_{1} C_{3}$ resonator blocks current at ${ }^{1} \mathrm{H}$ frequency. $C_{2}$ is chosen to resonate with $L$ close to ${ }^{13} \mathrm{C}$ frequency when

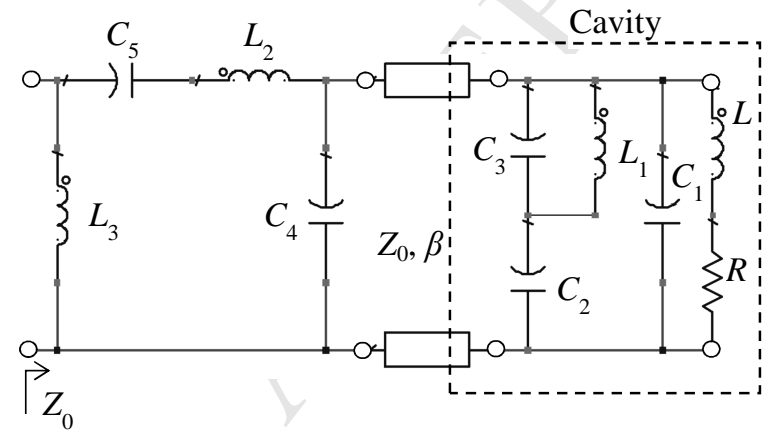

Figure 12. Schematic of the double resonance matching circuit for ${ }^{13} \mathrm{C}$ and ${ }^{1} \mathrm{H}$ operation. $C_{1}=15 \mathrm{pF}, C_{2}=180 \mathrm{pF}, C_{3}=30 \mathrm{pF}$, $C_{4}=1.6 \mathrm{pF}(2 \mathrm{pF}$ in series with $8.2 \mathrm{pF}), C_{5}=9.1 \mathrm{pF}, L_{1}=10 \mathrm{nH}$, $L_{2}=297 \mathrm{nH}, L_{3}=45 \mathrm{nH}$. connected in parallel to $C_{1}$. A short transmission line then leads to a circuit outside the cavity. For convenience of final adjustment and better flexibility, the rest of the circuit consists of two components for each operating frequency. At high frequency, the tuning and matching is realized using a low pass circuit consisting of $L_{2}$ and $C_{4}$. Due to low-pass behavior, the circuit is almost transparent for the low operating frequency $\left({ }^{13} \mathrm{C}\right)$. Following the same strategy, low frequency tuning and matching is realized using a high-pass circuit consisting of $L_{3}$ and $C_{5}$. This high-pass circuit is almost transparent for the high operating frequency $\left({ }^{1} \mathrm{H}\right)$. Choosing components in the way described above gives a good starting point for the design. The final circuit can then be fine-tuned and optimized using a circuit simulator, which will also allow to include parasitics of interconnects and lumped components as well as printed circuit board layout.

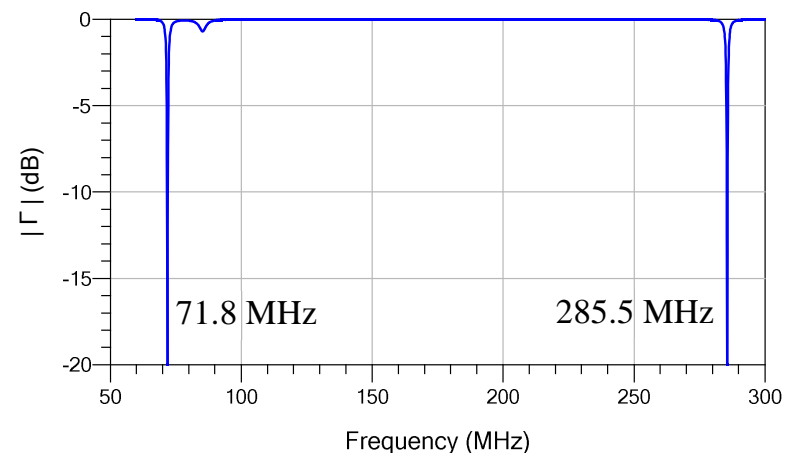

Figure 13. Magnitude of the reflection for the double resonance matching network for ${ }^{13} \mathrm{C}$ and ${ }^{1} \mathrm{H}$ cross polarization experiments shown in Figure 12. 
The response of the double resonance matching circuit is shown in Figure 13. As can be observed, matching occurs at the two design frequencies.

\section{Material and methods}

\subsection{Sample preparation}

The sample used for studying the $\mathrm{CP}$ efficiency consisted of $50 \mathrm{mM}$ TEMPOL (4-hydroxy-2,2,6,6- tetramethylpiperidin-1-oxyl), $4.5 \mathrm{M}{ }^{13} \mathrm{C}$-urea and $\mathrm{d}_{8}$ glycerol, $\mathrm{D}_{2} \mathrm{O}$, and $\mathrm{H}_{2} \mathrm{O}$ in the volumetric ratio $5: 2: 3$ for a labile proton ratio of ca. $54 \%$. In the sample composition study, the $\mathrm{D}_{2} \mathrm{O}$, and $\mathrm{H}_{2} \mathrm{O}$ ratios used were 5:0, 4:1, 2:3, and 0:5 (all chemicals from Sigma-Aldrich, except for the $\mathrm{d}_{8}$-glycrol from Cambridge Isotope Laboratories).

Sample preparation: TEMPOL and urea were weighted. Glycerol, $\mathrm{D}_{2} \mathrm{O}$ and $\mathrm{H}_{2} \mathrm{O}$ were added by pipet. The powders were dissolved by vortex and ultrasound. $50 \mu \mathrm{L}$ were transferred to the DNP vial and frozen in the polarizer. After sample cool down,

a)
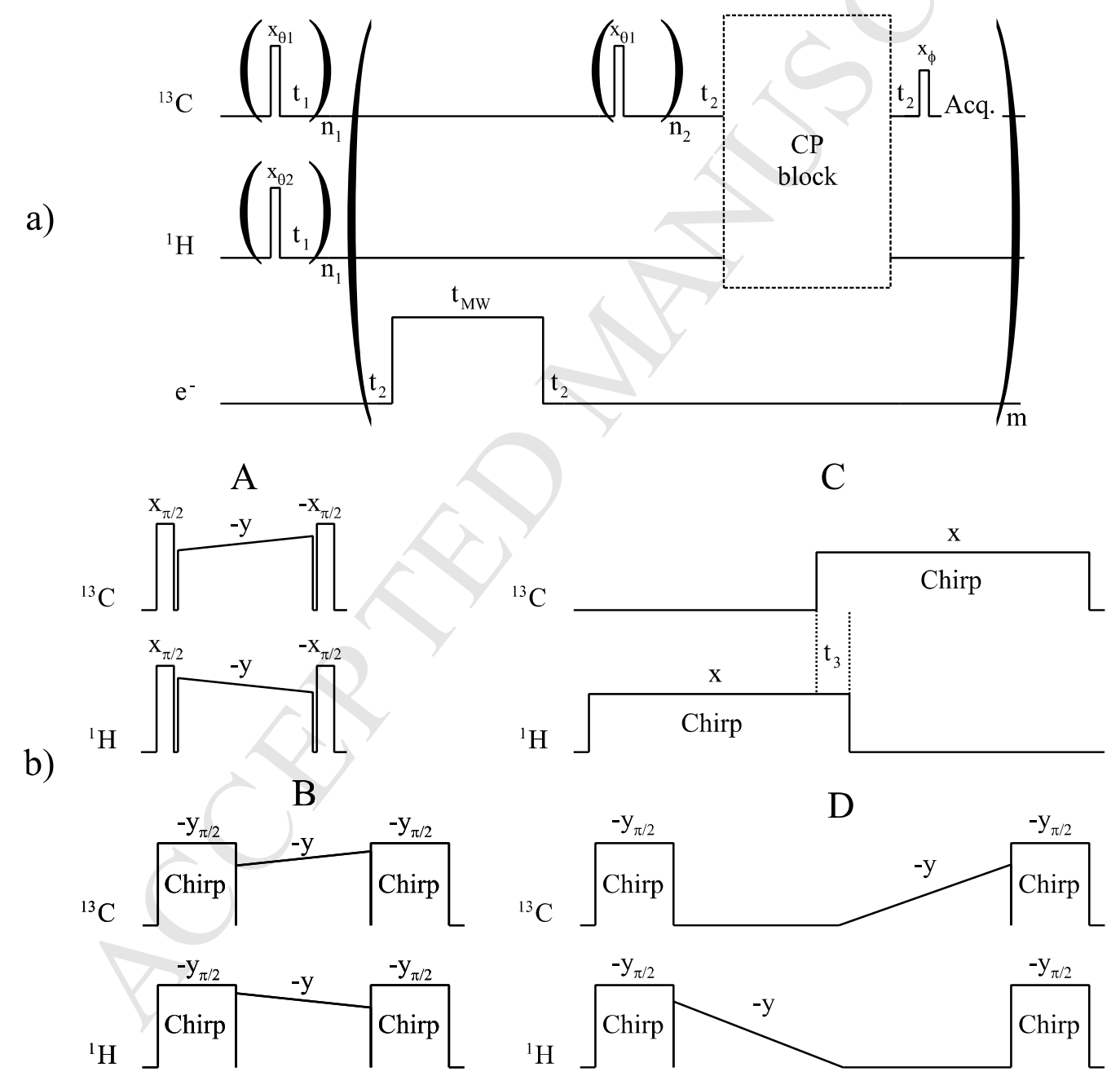

Figure 14. Pulse sequences used for testing CP efficiency. a) Overall testing sequence. b) The four different CP elements: A Hartmann-Hahn CP using hard 90-pulses, B Hartmann-Hahn CP using adiabatic half-chirp 90-pulses, C LAFDR, and D ADRF-ARRF. On top of pulses the phase and flip-angle of the pulse are indicated. Delays are indicated as well. $\mathrm{t}_{\mathrm{MW}}$ indicates the length of the build-up period in which microwaves are gated on. For successive (suc.) transfers $\mathrm{m}>1$ and $\mathrm{n}_{2}=0$ otherwise $\mathrm{m}=1$ and $\mathrm{n}_{2}=\mathrm{n}_{1}=1000 . \theta 1 \sim 21^{\circ}, \theta 2 \sim 28^{\circ}$ and $\phi \sim 1^{\circ} . \mathrm{t}_{1}=0.1$ $m s$ and $t_{2}=1 \mathrm{~s}$. $t_{3}$, chirps, sweeps and matching parameters were optimized for transfer efficiency. 
the local tune and match was verified using a vector network analyzer and the resonance frequencies were fine-tuned using an external duplexer. Finally, the sample height was adjusted for yielding the highest signal intensity and the microwave frequency was optimized for proton signal intensity: $188.085 \mathrm{GHz}$ with $\pm 50 \mathrm{MHz}$ modulation amplitude at $1 \mathrm{kHz}$; ca. $56 \mathrm{~mW}$. The microwaves were gated on during DNP build-up and off otherwise for improved $\mathrm{CP}$ efficiency [7].

\subsection{Equipment}

The DNP experiments used a setup similar to the one introduced in [15] as described in [16]. The NMR console used was a Varian Direct-Drive (Palo Alto, CA, USA). The Allen-Bradley resistors used for helium level regulation were lifted just above the tuning and matching circuit such that the circuit should be covered by liquid helium. The temperature was kept at $1.25 \mathrm{~K}$ according to the helium vapor pressure.

\subsection{Cross-polarization sequences}

The CP sequences tested in this study were: Hartmann-Hahn CP (HHCP) [4] with linear CP profiles on both channels, Laboratory Frame De- and Remagnetisation (LAFDR) [17], [8], and Adiabatic Demagnetisation in the Rotating Frame - Adiabatic Remagnetisation in the Rotating Frame (ADRFARRF) [18]. The HHCP and ADRF-ARRF were combined with hard-90-pulses or half-Chirp (adiabatic) 90-pulses (optimized parameters: $\mathrm{B}_{1}\left({ }^{13} \mathrm{C}\right) \sim 20 \mathrm{kHz}, \quad \mathrm{B}_{1}\left({ }^{1} \mathrm{H}\right) \sim 30 \mathrm{kHz}$, pulse length $250 \mu \mathrm{s}$, and frequency sweep $100 \mathrm{kHz}$ ). Furthermore, the HHCP sequences were tested with successive (suc.) transfers as well as single transfers. Pulse lengths, amplitudes, sweeps, offsets, and delays between successive transfers were optimized. The pulse sequences are shown in Figure 14.

\subsection{Double-resonance probe}

The double resonance tuning and matching circuit is designed to transform impedance of the coil to $50 \Omega$ at $71.8 \mathrm{MHz}\left({ }^{13} \mathrm{C}\right)$ and $285.5 \mathrm{MHz}\left({ }^{1} \mathrm{H}\right)$. The circuit is then connected with the interface on the

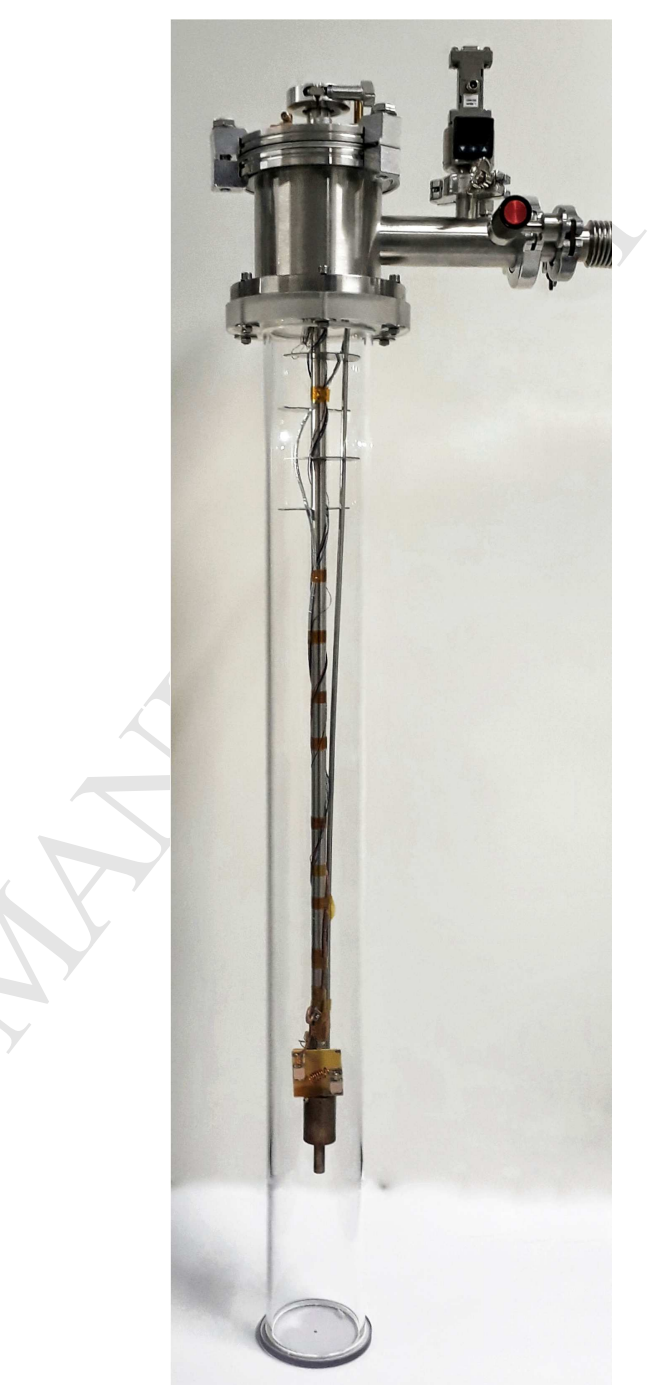

Figure 15. Photograph of the transparent plastic chamber for electrical discharge testing. On top the adapter for probe and pump connection is seen. The probe is inserted in the chamber.

flange of the probe using a standard $50 \Omega$ coaxial cable. The cable is equipped with an LC trap for ${ }^{13} \mathrm{C}$ frequency and "bazooka" trap for ${ }^{1} \mathrm{H}$ frequency to suppress common mode currents [19]. The ${ }^{13} \mathrm{C}$ and ${ }^{1} \mathrm{H}$ signals can then be separated outside the magnet by a duplexer containing a circuit with trimming capacitors for eventual fine-tuning.

The local tuning and matching circuit was constructed using home-build inductors and Passive Plus Inc. ceramic $1111 \mathrm{C}, 2225 \mathrm{C}$, and 3838C capacitors (Huntington, NY, USA). 
A chamber made of a transparent plastic tube (polycarbonate) was constructed for bench tests of the probe in helium atmosphere at variable pressure (Figure 15). The setup made direct observation of electrical discharges possible and eased the localization of the exact origin of these by varying both pressure and pulse amplitude.

\section{Results and Discussion}

\subsection{B $B_{1}$-field strengths and electrical discharge limits}

The $\mathrm{B}_{1}$-fields were measured by successive pulsing towards saturation of the resonance and fitting of the data to $k \cdot \cos ^{n}(\theta) ; n$ being the pulse number, $\theta$ the pulse flip angle and $k$ a constant. In Table 1 the maximum stable $B_{1}$-fields for $2 \mu$ s pulse trains are tabulated. Taking the coil geometry into consideration, very strong $\mathrm{B}_{1}$-fields are achieved, and supposedly sufficient for cross-polarization. However, considering the pulse lengths needed for cross-polarization, electrical discharges still occurs at somewhat lower amplitudes, as given in Table 2 for pulse lengths relevant for HHCP and LAFDR/ADRFARRF. The efforts to decrease the voltages by design of the circuit were focused on the ${ }^{13} \mathrm{C}$-channel, since this channel yields the lowest maximum $\mathrm{B}_{1}$-field, whereby it determines the maximum HHCP $\mathrm{B}_{1}$-field (due to the Hartmann-Hahn matching condition). For

Table $1 . \mathrm{B}_{1}$-field strengths determined using the saturation pulse method.

\begin{tabular}{cccc}
\hline \multicolumn{2}{c}{${ }^{1} \mathrm{H}$} & \multicolumn{2}{c}{${ }^{13} \mathrm{C}$} \\
\hline $\mathrm{P}$ & $\mathrm{B}_{1}$ & $\mathrm{P}$ & $\mathrm{B}_{1}$ \\
$(\mathrm{~W})$ & $(\mathrm{kHz})$ & $(\mathrm{W})$ & $(\mathrm{kHz})$ \\
\hline 33 & 44 & 199 & 40 \\
\hline
\end{tabular}

Table 2. Electrical discharge limits determined as unstable or increasing reflected power

\begin{tabular}{ccccc}
\hline & \multicolumn{2}{c}{${ }^{1} \mathrm{H}$} & \multicolumn{2}{c}{${ }^{13} \mathrm{C}$} \\
\hline $\begin{array}{c}\text { pulse } \\
\text { length } \\
(\mathrm{ms})\end{array}$ & $\mathrm{P}$ & $\mathrm{B}_{1}$ & $\mathrm{P}$ & $\mathrm{B}_{1}$ \\
$(\mathrm{~W})$ & $(\mathrm{kHz})$ & $(\mathrm{W})$ & $(\mathrm{kHz})$ \\
\hline 1 & 26 & 38 & 93 & 29 \\
20 & 4 & 18 & 9 & 8 \\
\hline
\end{tabular}

LAFDR (and ADRF-ARRF) one is free to optimize the amplitudes individually and an optimum $\mathrm{B}_{1}$-field for protons is found to be less than the maximum power. On the other hand, the ${ }^{13} \mathrm{C} \quad \mathrm{B}_{1}$-field optimization for LAFDR is limited by electrical discharges at $8 \mathrm{kHz}$ (optimization data not shown).

\subsection{Performance comparison of different $C P$ sequences}

The three different $\mathrm{CP}$ sequences (HHCP, LAFDR, and ADRF-ARRF) were optimized and compared for final ${ }^{13} \mathrm{C}$ polarization levels (Figure 16) and build-up times (Figure 17). The LAFDR was tested both with and without an overlap of the pulses $\left(\mathrm{t}_{3}\right.$ in Figure 14) and the HHCP was tested with hard and adiabatic 90 degree pulses. Moreover, successive transfers were tested for HHCP (suc. in Figure 16 and Figure 17).

At the given power levels HHCP and LAFDR performs equally, meaning that the $\mathrm{B}_{1}$-field strength is the limiting factor for HHCP according to [8]. Another signifier of $\mathrm{B}_{1}$-limiting performance is seen when comparing the HHCP with hard and half-chirp 90 degree pulses. The chirp is more efficient, especially with successive transfers. However, the improvement observed going from a hard to an adiabatic 90 degrees pulse possibly indicates $\mathrm{B}_{1}$ inhomogeneities over the sample volume as well. ADRF-ARRF was outperformed by the other CP

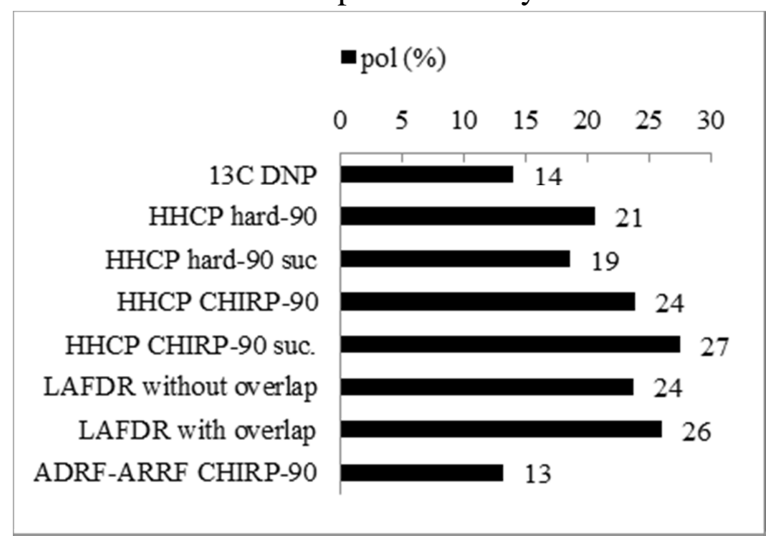

Figure 16. Comparison of solid-state ${ }^{13} \mathrm{C}$ polarization percentages achieved by $\mathrm{CP}$ sequences and by direct ${ }^{13} \mathrm{C} \mathrm{DNP} .{ }^{13} \mathrm{C}$-Urea-sample. $29 \mathrm{kHz} \mathrm{B}_{1}$ for HHCP and $8 \mathrm{kHz}$ for the longer LAFDR and ADRFARRF (max). ${ }^{1} \mathrm{H}$ polarization was determined to $74 \%$. 


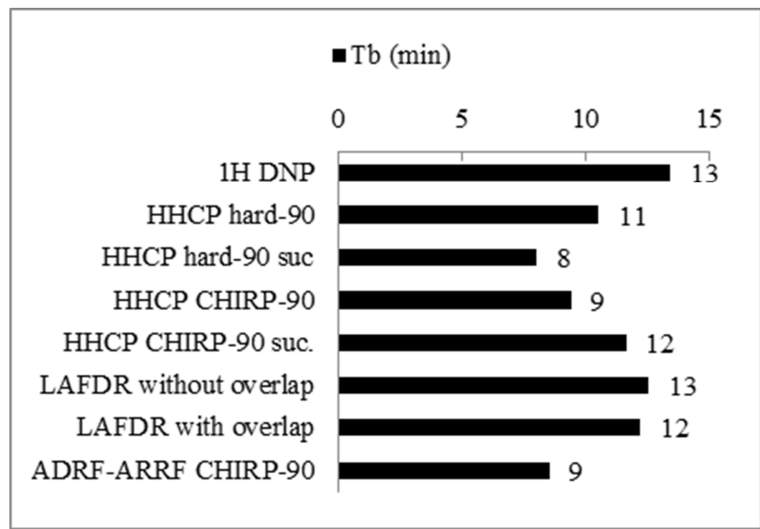

Figure 17. Comparison of solid-state ${ }^{13} \mathrm{C}$ polarization build-up time constants achieved by $\mathrm{CP}$ sequences with direct ${ }^{1} \mathrm{H}$ DNP. ${ }^{13} \mathrm{C}$-Ureasample. $29 \mathrm{kHz} \mathrm{B}_{1}$ for $\mathrm{HHCP}$ and $8 \mathrm{kHz}$ for the longer LAFDR and ADRF-ARRF (max). Direct ${ }^{13} \mathrm{C}$ DNP build-up showed a

characteristic time constant of $53 \mathrm{~min}$.

sequences.

The performances are in line with the DNP CP performances of dissolution capable setups described in the introduction with the exception of those presented in [7] - despite thorough efforts to improve the $\mathrm{CP}$ efficiencies. We ascribe the performance difference to the differences of coil geometry, sample formulation, and possibly sample heating.

\subsection{Proton-deuterium ratio in sample}

The impact of diluting the proton concentration was tested, since the transfer efficiency of $\mathrm{CP}$ at low $\mathrm{B}_{1}$-field strengths should be inhibited mostly by the strong dipolar couplings between protons. Samples with 4 different ratios of labile proton/deuterium were tested for $\mathrm{CP}$ performance using both HHCP and LAFDR at 11 and $6 \mathrm{kHz}$, respectively. The ratios between transferred and direct ${ }^{13} \mathrm{C}$ DNP are depicted in Figure 18. It is found that the increase in proton concentration lowers the efficiency of transfer especially for HHCP. For LAFDR the increase in total proton polarization (by increase of the size of the proton reservoir) counteracts the decreased $\mathrm{CP}$ efficiency. Increasing the proton concentration comes with the downside of decreased ${ }^{1} \mathrm{H}$ DNP build-up rates from $4 \mathrm{~min}$ at $31 \%$ to $14 \mathrm{~min}$ at $55 \%$

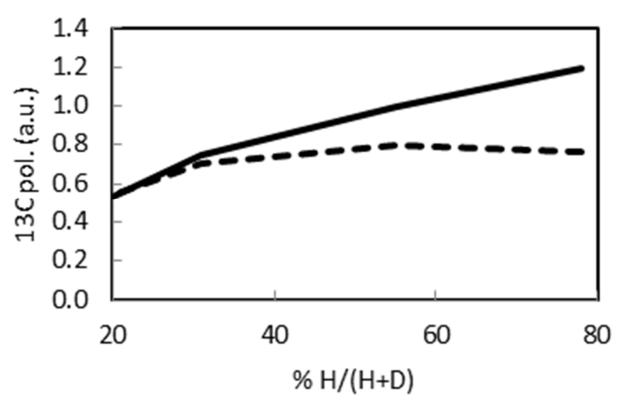

Figure 18. ${ }^{13} \mathrm{C}$ polarizations: Ratio of CP-DNP and direct DNP polarization as function of protonation degree of the sample. $\mathrm{HHCP}$ at $11 \mathrm{kHz}$ dashed line and LAFDR at $6 \mathrm{kHz}$ full line.

protonation (data not shown), speaking in favor of moderate degrees of protonation.

\section{Conclusions}

In order to minimize voltage amplitudes in the RF circuit the following should be considered:

Minimize the reactance of the coil. The voltage across the coil is directly proportional the magnitude of the coil impedance. Since, typically, the loss in the coil is quite low, it is the reactive part of the coil impedance which defines the total voltage. On the other hand, lower coil reactance makes the voltage (in Case 2 and Case 3) more dependent on transmission line length, since reactance of the transmission line becomes more dominant.

- The resistive part of the coil is usually not a design criterion and should, typically, be minimized for better system efficiency (yielding higher $\mathrm{B}_{1}$-fields). Larger resistive part, on the other hand, makes it easier to match to higher system impedance, like $50 \Omega$, for example. As a result, it also leads to lower voltage in a series capacitor.

- Local matching is optimal in terms of circuit voltage and efficiency. However, if local matching is not feasible due space constraints, quasi-distributed tuning and matching can be a good compromise, which delivers almost the same performance (if the cable length is 
relatively short), but allows for more flexibility.

Based on the described strategies, a CP-probe has been developed with resulting $\mathrm{B}_{1}$-field strengths of $44 \mathrm{kHz}$ and $40 \mathrm{kHz}$ for ${ }^{1} \mathrm{H}$ and ${ }^{13} \mathrm{C}$, respectively. $\mathrm{HHCP}$ is achieved at $\mathrm{B}_{1}=29 \mathrm{kHz}$, which enables successive transfers yielding $27 \%$ ${ }^{13} \mathrm{C}$ polarization with a characteristic build-up time of $12 \mathrm{~min}$. That is 2 times the direct ${ }^{13} \mathrm{C}$ DNP polarization and 4.4 times faster. The build-up time corresponds to the direct ${ }^{1} \mathrm{H}$ DNP build-up time as expected for ${ }^{1} \mathrm{H}$ DNP CP.

Regarding sample composition, a moderate protonation degree is the best compromise between long ${ }^{1} \mathrm{H}$ DNP build-up time (high protonation) and moderate ${ }^{1} \mathrm{H}$ dipolar couplings (diluted spin system, low protonation) impeding CP efficiency.

\section{Acknowledgments}

Acknowledgment for funding to the Danish National Research Foundation (DNRF124), Innovation Fund Denmark, and the European Union's Horizon 2020 research and innovation programme under the Marie Sklodowska-Curie grant agreement No. 642773 (EUROPOL)

\section{Appendix A.}

Here we derive equations for calculation of capacitors in Case 2, Figure 6. a.

First, we express the impedance at the input terminals of the transmission line section using transmission line theory [13]

$$
Z_{c}=Z_{0} \frac{Z_{\text {out }}+j Z_{0} \tan (\beta l)}{Z_{0}+j Z_{\text {out }} \tan (\beta l)}=Z_{0} \frac{\frac{R+j \omega L}{\mathfrak{B}_{2}(j R-\omega L)+1}+j Z_{0} \tan (\beta l)}{Z_{0}+j \frac{R+j \omega L}{\mathfrak{B}_{2}(j R-\omega L)+1} \tan (\beta l)}
$$

In order to match to $50 \Omega$, the following condition should be satisfied

$$
\operatorname{Re}\left\{Z_{c}\right\}=50
$$

The equation (A.2) is then solved with regard to $\mathfrak{B}_{2}$ assuming $Z_{0}=50 \Omega$. This results in eq. (9) in the main text.

The imaginary part of $Z_{\mathrm{c}}$ is compensated by the reactance of the series capacitor $X_{2}$ :

$$
\operatorname{Im}\left\{Z_{c}\right\}+X_{2}=0
$$

which, in combination with eq. (A.1), leads to expression (10) in the main text.

\section{References}

[1] J. Ardenkjær-Larsen, S. Bowen, J. R. Petersen, O. Rybalko, M. S. Vinding, M. Ullisch and N. C. Nielsen, Magn Reson Med., vol. 81, pp. 2184-2194, 2019.

[2] A. Capozzi, S. Patel, C. P. Gunnarsson, I. Marco-Rius, A. Comment, M. Karlsson, M. H. Lerche, O. Ouari and J. H. Ardenkjær-Larsen, Angew. Chem. Int. Ed., vol. 58, p. 1334, 2019.

[3] J.-N. Dumez, J. Milani, B. Vuichoud, A. Bornet, J. Lalande-Martin, I. Tea, M. Yon, M. Maucourt, C. Deborde, A. Moing, L. Frydman, G. Bodenhausen, S. Jannin and P. Giraudeau, Analyst, vol. 140, p. 5860, 2015.

[4] S. R. Hartmann and E. L. Hahn, Phys. Rev., vol. 128, pp. 2042-2053, 1962.

[5] S. Jannin, A. Bornet, S. Colombo and G. Bodenhausen, Chem Phys Lett, vol. 517, pp. 234-236, 2011.

[6] M. Batel, M. Krajewski, A. Däpp, A. M. H. Hunkeler, S. Kozerke and M. Ernst, Chem Phys Lett, vol. 554, pp. 72-76, 2012.

[7] A. Bornet, A. Pinon, A. Jhajharia, M. Baudin, X. Ji, L. Emsley, G. Bodenhausen, J. H. Ardenkjaer-Larsen and S. Jannin, Phys.Chem.Chem.Phys., vol. 18, pp. 3053030535, 2016.

[8] M. Batel, A. Däpp, A. Hunkeler, B. Meier, S. Kozerke and M. Ernst, Phys Chem Chem Phys, vol. 16, pp. 21407-21416, 2014. 
[9] A. J. Pérez Linde, "Application of cross polarisation techniques to dynamic nuclear polarisation dissolution experiments. $\mathrm{PhD}$ thesis," University of Nottingham, 2010.

[10] V. D. Kodibagkar and M. S. Conradi, J. Magn. Reson., vol. 144, pp. 53-57, 2000.

[11] J. Vidkjær, "RF Communication Circuits".

[12] D. Pozar, "Impedance Matching and Tuning," in Microwave Engineering, Wiley, 2004.

[13] D. Pozar, Microwave Engineering, 4th ed., Wiley, p. 732.

[14] A. Bornet, R. Melzi, A. J. P. Linde, P. Hautle, B. van den Brandt, S. Jannin and G. Bodenhausen, J. Phys. Chem. Lett., vol. 4, pp. 111-114, 2013.

[15] J. H. Ardenkjaer-Larsen, B. Fridlund, A. Gram, G. Hansson, L. Hansson, M. H. Lerche, R. Servin, M. Thaning and K. Golman, Nmr. Proc. Natl. Acad. Sci. U. S. A, vol. 100, p. 10158-10163, 2003.

[16] A. Capozzi, M. Karlsson, J. R. Petersen, M. H. Lerche and J. H. Ardenkjaer-Larsen, J. Phys. Chem. C, vol. 122, pp. 7432-7443, 2018.

[17] J.-S. Lee and A. K. Khitrin, J. Chem. Phys., vol. 114504, p. 128, 2008.

[18] A. G. Redfield, Science, vol. 164, pp. 10151023, 1969.

[19] J. T. Vaughan and J. R. Griffiths, RF Coils for MRI, : John Wiley \& Sons Ltd, 2012.

[20] S. Jannin, Chem. Phys. Lett., vol. 549, pp. 99102, 2012. 
In order to minimize voltage amplitudes in the RF circuit the following should be considered:

- Minimize the reactance of the coil

- The resistive part of the coil is usually not a design criterion and should, typically, be minimized for better system efficiency.

- If local matching is not feasible due space constraints, quasi-distributed tuning and matching can be a good compromise.

- $\quad$ Based on the described strategies, a CP-probe has been developed with resulting $\mathrm{B}_{1}$-field strengths of $44 \mathrm{kHz}$ and $40 \mathrm{kHz}$ for ${ }^{1} \mathrm{H}$ and ${ }^{13} \mathrm{C}$, respectively.

- $\mathrm{HHCP}$ is achieved at $\mathrm{B}_{1}=29 \mathrm{kHz}$, which enables successive transfers yielding $27 \%{ }^{13} \mathrm{C}$ polarization with a characteristic build-up time of $12 \mathrm{~min}$. 